\title{
Emociones en educación física desde la perspectiva constructivista: análisis de los currículos de España y Chile
}

Felipe Nicolás Mujica Johnson Doctorando en Ciencias de la Actividad Física y del Deporte Universidad Católica de Temuco Chile

fmujica@live.cl

https://orcid.org/0000-0002-6956-2357

Nelly del Carmen Orellana Arduiz Doctora en Filosofía y Educación Universidad de Playa Ancha - Chile norellan@upla.cl

https://orcid.org/0000-0002-5742-4539

Artículo de Reflexión

Recepción: 9 de enero de 2019

Aprobación: 1 de junio de 2019

https://doi.org/10.19053/22160159.v10.n25.2019.8468

\section{Resumen}

Las principales teorías del aprendizaje constructivista reconocen la dimensión emocional como un componente esencial del acto educativo, ya que es uno de los elementos mediadores en la interpretación y significación de la realidad. Esta perspectiva ha impactado en todas las disciplinas del currículo y en específico en la educación física, que es una asignatura bastante especial, ya que exige a los educandos involucrarse en la totalidad de sus dimensiones, en donde además la exposición social suele ser una situación muy común. Ante este escenario, se han analizado elementos de los currículos español y chileno, con la finalidad de describir la incorporación y sistematización de las emociones, entendiendo que esta medida es fundamental para garantizar una educación integral. A partir de este análisis, se puede indicar que la legislación educativa española ha sistematizado diferentes competencias 
que incluyen aquella dimensión, mientras que la legislación chilena ha incorporado objetivos actitudinales que asocian la dimensión afectiva, pero no se aprecia una programación rigurosa. Por lo tanto, se concluye que existe una necesidad de abrir un debate en torno a la educación integral en Chile.

Palabras clave: teoría de la educación, educación formal, afectividad, innovación pedagógica, entorno cultural

\section{Emotions in physical education from the constructivist perspective: analysis of the curriculums of Spain and Chile}

\section{Abstract}

The main theories of constructivist learning recognize the emotional dimension as an essential component of the educational act, since it is one of the mediating elements in the interpretation and meaning of reality. This perspective has impacted all the disciplines of the curriculum and specifically that of Physical Education, which is a very special subject, since it requires learners to be involved in all its dimensions, where in addition the social exposure is usually a very common situation. Given this scenario, elements of the Spanish and Chilean curriculums have been analyzed, with the aim of describing the incorporation and systematization of emotions, understanding that this measure is fundamental to guarantee a comprehensive education. From this analysis, it can be said that the Spanish educational legislation has systematized different competences that include that dimension, while the Chilean legislation has incorporated attitudinal objectives that include the affective dimension, but no rigorous programming is appreciated. Therefore, it is concluded that there is a need to open a debate on integral education in Chile.

Keywords: theory of education, formal education, affectivity, pedagogical innovation, cultural environment

\section{Émotions dans l'éducation physique du point de vue constructiviste : analyse du programme d'études de l'Espagne et du Chili}

\section{Résumé}

Les principales théories de l'apprentissage constructiviste reconnaissent la dimension émotionnelle comme une composante essentielle de l'acte 
éducatif, car c'est l'un des éléments médiateurs de l'interprétation et du sens de la réalité. Cette perspective a eu un impact sur toutes les disciplines du programme d'études et en particulier celle de l'éducation physique, qui est une matière très spéciale, car elle exige que les apprenants soient impliqués dans toutes ses dimensions, où l'exposition sociale est en outre une situation très courante. Dans ce scénario, des éléments du programme d'études espagnol et chilien ont été analysés, dans le but de décrire l'incorporation et la systématisation des émotions, sachant que cette mesure est fondamentale pour garantir une éducation complète. Il ressort de cette analyse que la législation éducative espagnole a systématisé différentes compétences englobant cette dimension, tandis que la législation chilienne a incorporé des objectifs comportementaux associant la dimension affective, mais aucune programmation rigoureuse n'est appréciée. Par conséquent, il est conclu qu'il est nécessaire d'ouvrir un débat sur l'éducation intégrale au Chili.

Mots-clés : théorie de l'éducation, éducation formelle, affectivité, innovation pédagogique, environnement culturel

\section{Emoções em educação física desde a perspectiva construtivista: análise dos currículos na Espanha e no Chile}

\section{Resumo}

As principais teorias da aprendizagem construtivista reconhecem a dimensão emocional como um componente essencial do ato educativo, já que é um dos elementos mediadores na interpretação e significação da realidade. Esta perspectiva tem impactado em todas as disciplinas do currículo, e em específico na educação física, que é uma matéria bastante especial, já que exige aos educandos envolver-se na totalidade de suas dimensões, em onde ademais a exposição social costuma ser uma situação muito comum. Ante este cenário, têm-se analisado elementos dos currículos espanhol e chileno, com a finalidade de descrever a incorporação e sistematização das emoções, entendendo que esta medida é fundamental para garantir uma educação integral. A partir desta análise, pode-se indicar que a legislação educativa espanhola tem sistematizado diferentes competências que incluem aquela dimensão, enquanto a legislação chilena tem incorporado objetivos atitudinais que associam a dimensão afetiva, mas não se aprecia uma programação rigorosa. Por conseguinte, conclui-se que existe uma necessidade de abrir um debate em torno da educação integral no Chile.

Palavras-chave: teoria da educação, educação formal, afetividade, inovação pedagógica, meio cultural 


\section{Introducción}

Las instituciones de educación formal se caracterizan por responder a la legislación vigente de cada Estado, que en una sociedad democrática significa el resultado de una construcción política. Entonces, la educación formal ha de ser entendida como "aquella que concluye con titulaciones reconocidas y otorgadas según las leyes educativas promulgadas por los Estados, desde los diplomas de enseñanza primaria hasta la titulación de doctor" (Colom, 2005, p. 11). A partir de esta aclaración, se comprende que la educación formal "esencialmente es una práctica social, epistemológica y política, que compromete a sus protagonistas, en cualquiera de sus posiciones" (Rivas, Leite \& Cortés, 2014, p. 13). Por lo mismo, es fundamental comprender las teorías implícitas en las legislaciones educativas, ya que en ningún caso representan una neutralidad ideológica y epistemológica (Moreno, Rivera \& Trigueros, 2014). De esta forma, se podría señalar que la presencia obligatoria de la educación física en el currículo educativo de las diferentes naciones es una decisión temporal, que podría cambiar o mantenerse según las perspectivas educativas que predominen tanto en la sociedad civil, como en la académica, y por supuesto, en el entorno político. En relación con lo anterior, la baja presencia que tendría la educación física en el currículo escolar de Latinoamérica como en el de España en comparación con otras asignaturas, estaría fundado en motivos ideológicos que han dominado la discusión educativa. Esta situación se encuentra muy bien reflejada en un informe que describe la educación física en los centros escolares de Europa (Comisión Europea/EACEA/Eurydice, 2013), el cual ubica a España entre los últimos países en función de las horas lectivas dedicadas a esta asignatura. Sin embargo, las horas lectivas en España serían solo una de las varias problemáticas en torno a la asignatura, lo que lleva a concluir que "las políticas en materia de Educación Física van en una dirección contraria a las directrices y recomendaciones internacionales" (Gambau, 2015, p. 67).

Por consiguiente, para garantizar una perspectiva actualizada de los procesos educativos en los países democráticos, es conveniente mantener una constante comunicación entre el ámbito académicocientífico y el ámbito político, debido a que son los protagonistas de este último entorno los que toman las decisiones más importantes en las áreas fundamentales de la vida social, como lo es la educación. 
En resumen, se ha de comprender que la actividad educativa de carácter formal no corresponde a una actividad propiamente científica, sino que más bien puede ser orientada por el conocimiento de la ciencia, pero pertenece a una actividad esencialmente política de cada Estado. En relación con este planteamiento, la esencia de la educación estaría muy bien representada en las siguientes ideas:

La educación es esencialmente el modo de vida que muestra la forma de ser, pensar y actuar de cada hombre, de cada pueblo, de cada época. Es realización histórico-cultural, en cuanto que la educación informa, promueve, orienta los procesos culturales imprimiéndoles su razón de ser y de acontecer (Capitán, 2002, p. 7).

A pesar de que los centros de escolarización correspondan a un espacio de educación formal, son a la vez un espacio que interactúa a diario con la educación no formal y con la educación informal. La educación no formal es una actividad educativa organizada y sistemática, que se realiza fuera de un marco oficial del Estado, como es el caso de un club deportivo; mientras que la educación informal es aquella que duraría toda la vida y se compone de las experiencias cotidianas con las amistades, la familia o en la vida sociocomunitaria de los diferentes pueblos o ciudades (Cabalé \& Rodríguez, 2017).

Por medio de una breve revisión de la literatura, es posible identificar que una perspectiva que dominaba el ámbito social de la educación hasta 1970 era el funcionalismo, que se enmarca en una visión positivista de la realidad, hasta que en esa misma época irrumpió en las ciencias sociales la perspectiva interpretativa que produjo un nuevo panorama epistemológico (Carr \& Kemmis, 1988). Aquella nueva perspectiva, señalaba que la sociedad no era un sistema independiente de las personas, sino que la "característica crucial de la realidad social es la posesión de una estructura intrínsecamente significativa, construida y sostenida por las actividades interpretativas rutinarias de sus miembros individuales" (Carr \& Kemmis, 1988, p. 99). De esta forma, se posiciona una idea contraria a la objetividad independiente de la sociedad, al argumentar que "la sociedad posee cierto grado de objetividad gracias a que los actores sociales, en el proceso de interpretación de su mundo social, la exteriorizan y objetivan" (Carr \& Kemmis, 1998, p. 99). En cuanto a la principal función social de la 
escuela, las diferentes corrientes de la sociología indican que sería "la preparación de los individuos de las nuevas generaciones para su incorporación futura al mundo del trabajo" (Pérez, 1999, p. 19). No obstante, la escuela, al ser una institución social compuesta por una diversidad de interpretaciones, contradicciones y problemáticas, genera que el proceso de socialización siga un trayecto no lineal, debido a su complejidad.

De esta forma, en los centros educativos el proceso se realiza a través "de un complicado y activo movimiento de negociación donde las reacciones y resistencias de profesores/as y alumnos/as como individuos o como grupos pueden llegar a provocar el rechazo o ineficacia de las tendencias reproductoras de la institución escolar" (Pérez, 1999, p. 24). Considerando aquellas características particulares que acompañan y definen en un sentido históricocultural a los estudiantes y a los docentes durante el proceso educativo, se ha creado el concepto del currículo oculto, que representa la realidad que escapa de las delimitaciones establecidas en el currículo formal. En este concepto, se incluyen tanto "aspectos educativos sobre los cuales no incide la institución escolar educación extraescolar- cuanto actuaciones surgidas dentro de la propia institución pero que no aparecen referenciados en el currículum manifiesto, sea porque son inconscientes, sea porque se pretenden ocultar realmente" (Sarramona, 2008, p. 157).

Por otro lado, la discusión teórica en torno a la aplicación del campo psicológico al pedagógico ha aportado variadas teorías constructivistas sobre el aprendizaje que permitieron trascender la pedagogía conductista. Para este trabajo es preciso destacar a David Ausubel -1918-2008-, por el aporte de su teoría sobre el aprendizaje significativo, en la cual se destaca la relevancia de las experiencias previas al aprendizaje y su interacción con la dimensión afectiva-emocional en los educandos (Viera, 2003). A su vez, es necesario también destacar a Lev Vygotsky -1896-1934-, quien postuló el origen social de la inteligencia (Echeita \& Martín, 1990). Este autor, representante de la psicología soviética, señaló a diferencia de las teorías desarrolladas por la escuela de Ginebra, que la socialización no favorecía la inteligencia, sino que la originaba, debido a que "toda función superior siempre aparece primero en el plano interpersonal y después pasa al plano intrapersonal mediante un proceso de internalización, en el que desempeña un 
papel fundamental el lenguaje" (Echeita \& Martín, 1990, p. 51). Por lo tanto, uno de los conceptos fundamentales de su teoría es el de la interiorización, el cual explica el autor así:

Cualquier función en el desarrollo cultural del niño aparece dos veces, o en dos planos diferentes. En primer lugar aparece en el plano social y después en el plano psicológico. En principio aparece entre las personas como una categoría interpsicológica, y después aparece en el niño comouna categoría intrapsicológica. Esto es igualmente cierto con respecto a la atención voluntaria, la formación de conceptos y el desarrollo de la volición (Vygotsky, 1979, p. 94).

Con base en este conocimiento, el ser humano construye su personalidad en las relaciones sociales caracterizadas por un específico medio ambiente (Vygotsky, 2000), de modo que para poder comprender las situaciones sociales, es fundamental atender a los aspectos histórico-culturales que determinan el ambiente y a su vez, se encuentran asociados a la configuración subjetiva-emocional del alumnado (González, 2009).

La relevancia de estas perspectivas académicas han logrado bastantes progresos en los currículos educativos de algunos países, como es el caso de España, pero todavía sigue siendo un tema que debe ser debatido y ajustado a la investigación pedagógica (Jordi \& Torrebadella, 2018; Méndez, Fernández-Río, Méndez \& Prieto, 2015; Vicente, 2016). Sin embargo, en los países latinoamericanos como Chile se puede apreciar un menor avance en estas materias (Moreno, 2018). Por lo tanto, a través de este artículo se pretende fundamentar la importancia de las emociones en los procesos educativos bajo la perspectiva constructivista. En una primera parte se describen las ideas de los principales teóricos del constructivismo, mientras que en la segunda parte se exponen los alcances curriculares que ha tenido esta teoría en la educación física española, para finalizar con un análisis curricular en torno a la educación física chilena.

\section{Educación constructivista}

La educación constructivista emerge para reivindicar la complejidad del acto educativo, al entender que no se puede mantener una reducción lineal de causa y efecto para orientar el aprendizaje. Esta perspectiva "entiende que el proceso de enseñanza 
y aprendizaje se vertebra a partir de las relaciones conjuntas entre el profesor y el alumno alrededor del contenido" (López-Ros, Pradas \& Font, 2014, p. 34). Con respecto al rol del alumnado en esta visión epistemológica, se entiende que "el alumno no solo posee unos conocimientos previos sobre muchos de los conceptos básicos de la ciencia escolar, sino que estos juegan un papel de primera magnitud en la adquisición de nuevos conocimientos" (Perales, 2000, p. 34). Para representar la concepción constructivista del aprendizaje, se afirma que su principal idea "es que el sujeto 'construye' el conocimiento mediante la interacción que sostiene con el medio social y físico" (Sarramona, 2008, p. 249).

Esta perspectiva ha recurrido a diversas teorías psicológicas como se mencionaba anteriormente, entre las que destaca Piaget, Vygostsky y Ausubel (Perales, 2000; Sarramona, 2008). Piaget realiza importantes aportes al aprendizaje activo, pero uno de los aspectos débiles de su teoría es la poca consideración del entorno social que engloba el aprendizaje. Por lo mismo, existen discrepancias entre Piaget y Vygotsky. Una de ellas es en cuanto a los conceptos de aprendizaje y desarrollo, ya que para Vygotsky estos conceptos no son coincidentes porque "existe un desarrollo efectivo y un desarrollo potencial, y señala que la enseñanza debe dirigirse al segundo para optimizar todas las posibilidades del educando" (Sarramona, 2008, p. 250). En torno al ámbito social, la naturaleza del lenguaje fue otro punto de discrepancia, de modo que a diferencia de Piaget, "Vygotsky considera el lenguaje como un fenómeno social, que sirve para la comunicación con las personas que rodean al niño antes de que este sea capaz de interiorizarlo para su comunicación intrapersonal" (Sarramona, 2008, p. 250).

Por otra parte, en la teoría del aprendizaje significativo, "Ausubel propone un aprendizaje a partir de los saberes previos del aprendiz” (Germania \& García, 2017, p. 87). De esta forma, le otorga una gran importancia a la interacción del nuevo conocimiento con las experiencias previas al aprendizajey la dimensión afectiva-emocional del educando, "por lo que para Ausubel los nuevos significados son el producto del intercambio entre el material potencialmente significativo y la disposición subjetiva (emocional y cognitiva) del educando, modificándose esta última constantemente" (Viera, 2003, p. 38). Por lo mismo, enfatiza la necesidad de desarrollar una educación integral en el aula educativa, donde lo emocional 
y lo cognitivo sean igual de importantes. Sin embargo, esto no ha sido del todo bien aplicado en los currículos escolares, ya que "los programas y prácticas educativas perseveran en dar desmedida relevancia a la dimensión cognitiva en desmedro de la emocional" (Albornoz, 2009, p. 68). En cuanto a la aplicación de esta teoría en el contexto educativo, Ausubel "propone un aprendizaje progresivo y comprensivo [...] igualmente, la disposición positiva del sujeto que aprende está íntimamente relacionada con el material de aprendizaje que se presenta para el logro de un aprendizaje significativo" (Cáceres \& Munévar, 2016, p. 5).

Los postulados presentados en torno a la psicología y el aprendizaje, justifican la necesidad de estudiar la dimensión cognitiva-subjetiva de las emociones en el aula escolar, al considerar que existe una particularidad psicológica de cada persona dentro de un centro educativo y, en especial, dentro de un aula escolar - alumnado-profesorado-. Esa individualidad a la que se hace referencia no es inalterable por el entorno social y por las experiencias de vida, por lo que, para lograr una buena comprensión de ella, es indispensable considerar los sucesos histórico-culturales que acontecen en forma cotidiana y dinámica en la vida de las personas. En este sentido, es preciso entender que "las emociones se encuentran en una constante significación a medida que la persona participa activamente en los procesos de socialización" (MujicaJohnson \& Jiménez, 2019, p. 154).

Otro importante autor que contribuyó en forma notable a la teoría del constructivismo es Jerome Bruner -1915-2016-, reconocido por sus aportes a la psicología cognitiva, al considerar la dimensión cultural en el funcionamiento de la mente humana. Esta perspectiva difiere de la teoría que interpreta el funcionamiento de la mente humana desde una perspectiva computacional, debido a que "la participación del hombre en la cultura y la realización de sus potencialidades mentales a través de la cultura, hacen que sea imposible construir la psicología humana basándonos solo en el individuo" (Bruner, 1991, p. 28). El fundamento que inspira a la psicología cultural hace referencia al rol que tiene la cultura en la evolución de la mente humana, pues se considera que aquel desarrollo mental está asociado al progreso de un cierto estilo de vida, "en la que la «realidad» está representada por un simbolismo compartido por los miembros de una comunidad cultural en la que 
la forma de vida técnico-social es a la vez organizada y construida en términos de ese simbolismo" (Bruner, 1997, p. 21). Entre los planteamientos de Bruner (1989), se aprecia un distanciamiento con respecto a un determinismo cultural que niega el factor biológico, de modo que no hace parte de la discusión que enfrenta la herencia contra el medio. Ante esta discusión, plantea que "no hay ningún fenómeno psicológico sin un organismo que exista biológicamente, ni tampoco hay ningún fenómeno que tenga lugar fuera de un ambiente" (Bruner, 1989, p. 149). Por lo tanto, sostiene que ambas dimensiones son fundamentales en el desarrollo humano, por lo que, desde una perspectiva científica, sería posible estudiar la interacción del componente biológico con el del medio cultural y de esta forma "aprender qué tipo de peculiaridades culturales dan lugar a una diferencia intelectual en determinados momentos del desarrollo y cómo ocurre en concreto" (Bruner, 1989, p. 149).

Bruner fundamenta una perspectiva integrada perteneciente a la educación constructivista, lo cual se puede entender como un llamado a los profesionales que se desempeñan en el área pedagógica a no descontextualizar los procesos educativos. En cuanto a un análisis sobre la teoría psicocultural de Bruner en el contexto pedagógico, se ha indicado que este autor propone que "solo es posible comprender plenamente la actividad humana cuando esta se analiza sin prescindir del marco natural, social, cultural e histórico en el que dicha actividad está situada" (Lacasa, 1994, p. 24). De acuerdo con estos postulados, algunos estudios cuantitativos han considerado el historial deportivo como un factor cultural para comprender las emociones del alumnado en la clase de educación física (Duran, Lavega, Planas, Muñoz \& Pubill, 2014; Duran et al., 2015). Sin embargo, no se han logrado identificar estudios cualitativos que asocien el historial deportivo a los significados emocionales del alumnado.

\section{Constructivismo en la educación física de España}

Una educación física constructivista sostiene que el alumnado es el propio creador de su aprendizaje y el docente actúa como facilitador del proceso, al asumir un rol de guía o mediador pedagógico (Blández, 200o; Mujica-Johnson \& Jiménez, 2019). Además, el propósito de este enfoque sería generar aprendizajes motores que puedan ser significativos para el alumnado, lo cual 
requiere la presencia de algunos elementos esenciales. Entre ellos, para que se logre un aprendizaje significativo, es fundamental que en el alumnado "exista una aptitud y una actitud adecuadas, es decir, que esté capacitado para abordarlo y que esté abierto y predispuesto a realizarlo" (Blández, 2000, p. 19). Justamente, el tema de la actitud es clave para justificar la relevancia de las emociones en el alumnado durante las clases de educación física (Gil \& Martínez, 2016; Zamorano, Gil-Madrona, Prieto-Ayuso \& Zamorano, 2018), ya que la buena o mala actitud de una persona ante una situación u objeto está asociada a la percepción emocional, debido a que la actitud consta de un componente cognitivo, emocional y conductual (Bolívar, 1992). Para comprender mejor este segundo componente en particular, se puede decir que "el componente emocional de la actitud, se refiere a los sentimientos favorables o desfavorables, que experimenta la persona con relación al objeto de actitud" (Mula \& Navas, 2011, p. 18).

Por consiguiente, se desprende que las emociones positivas para el bienestar subjetivo favorecen la construcción de aprendizajes significativos, al contribuir en la actitud positiva de los aprendices, mientras que las emociones negativas generarían un efecto contrario. Cuando se reconocen estos significados actitudinales que median la actividad educativa, se debe considerar la importancia de la subjetividad humana en el proceso de enseñanza-aprendizaje. Al transferir este conocimiento a la educación física, se evidencia la necesidad de que el profesorado incluya el historial de la actividad motriz del alumnado en su análisis pedagógico, que se encuentra también asociado a su dimensión afectiva, porque "desde el punto de vista motriz, para una niña o un niño, un ambiente de aprendizaje tiene sentido si es capaz de plantearse la forma de resolverlo, y tiene significado si lo que percibe es suficientemente interesante" (Blández, 2000, p. 19).

El impacto de la perspectiva constructivista en la pedagogía del deporte se puede apreciar en el modelo de la enseñanza comprensiva en el deporte, el cual trasciende la mirada mecanicista o del cuerpo máquina que había predominado históricamente (López-Ros et al. 2014). Algunos conceptos relevantes de este modelo educativo son el aprendizaje y el rendimiento situado. En el aprendizaje no se reduce la comprensión del juego de los educandos al conocimiento 
específico de reglas o roles, sino que es "un concepto más amplio relativo al 'saber jugar' y que incluye la necesidad de conocer y ser capaz de organizar los diferentes roles de juego en relación con todos los aspectos relevantes que definen al mismo" (López, 2010, p. 44). En cuanto al rendimiento situado, este hace referencia a que las respuestas motrices durante la práctica deportiva deben ser interpretadas de acuerdo con su contexto concreto, porque los escenarios deportivos se diferencian unos de otros por sus características culturales, como también por los fines de la práctica (López, 2010; Mujica-Johnson \& Jiménez, 2019).

Este modelo, por su corriente epistemológica, considera la importancia de que los educandos produzcan aprendizajes significativos, por lo que es importante tener en cuenta la relación de los nuevos conocimientos de los educandos con los anteriores, ya que a partir de este proceso cognitivo se generaría un enriquecimiento y una nueva disponibilidad cognoscitiva (López, 2010). En cuanto a las actividades motrices, se podría analizar no solamente la relación del conocimiento cognitivo, sino que también la interacción de actividades motrices con las nuevas habilidades motrices específicas de cada deporte, de modo que si no existiese una relación, la actividad carecería de sentido para el educando. Esta situación que no contribuiría a un aprendizaje significativo podría estar sucediendo con los estudiantes que poseen un historial deportivo relacionado con una unidad didáctica específica, motivo por el cual se considera necesario que se desarrollen diferentes investigaciones en torno a ese escenario desde la perspectiva emocional (Zamorano et al., 2018). En el mismo sentido, se puede mencionar que las posibilidades educativas que ofrece el deporte aplicado a la educación física están sujetas a las "propuestas de participación, inclusión y con incidencia en las posibilidades motrices, cognitivas y socio afectivas del alumnado" (Castejón, 2010, p. 31).

A continuación se realizará un análisis del currículo español, ya que es uno de los países iberoamericanos que en la actualidad está desarrollando una educación por competencias y, además, es el país que presenta una notable mayoría de publicaciones relacionadas con las emociones en el contexto de educación física (Bermúdez \& Sáenz-López, 2019; Mujica, Orellana \& Concha, 2017), lo cual es fundamental para la discusión pedagógica. El currículo de educación física en España ha introducido importantes 
cambios epistemológicos desde la reforma educativa de 1990, que estableció los primeros pasos de una pedagogía constructivista. En 2006 se realizó otra importante innovación, que fue la inclusión de las competencias en la legislación educativa. Con respecto al significado del término competencia, Lleidà (2016), con base en la LOMCE (Ley Orgánica 8/2013), señala que hace referencia a "las capacidades para aplicar de forma integrada conceptos, habilidades, actitudes, emociones, motivaciones para dar respuesta a problemas y situaciones en diferentes contextos" (p. 25). Entre los aspectos que se han de destacar de la enseñanza por competencias, está la consideración de la formación integral del humano, ya que las competencias básicas "recogen la importancia de capacitar para el desarrollo de todas las dimensiones humanas, dando entre ellas un lugar importante a la dimensión afectiva como condición sine qua non para formar la formación integral del alumnado" (Pastor \& Cuevas, 2011, p. 47). Un aspecto clave en esta formación es que las competencias deben ser evaluadas sistemáticamente, para garantizar una "adquisición eficaz de las competencias y su integración efectiva en el currículo" (Boletín Oficial del Estado, 2014, p. 4).

Es preciso mencionar que una programación de aula desde una perspectiva constructivista no se puede reducir a una mera organización de aspectos que estarán presentes en el proceso pedagógico, sino más bien, debe ser entendida como un proceso abierto, flexible y dinámico, que incluya una reflexión constante, acompañado de una indagación sistemática que permita adaptar los elementos curriculares a las características y necesidades de cada contexto educativo (González \& Campos, 2014). Para mantener la idea de la autonomía del profesorado para situar su programación curricular, la interpretación personal del entorno educativo debe siempre respetar los aspectos básicos que establece la legislación educativa (Pastor \& Cuevas, 2011). Cada una de estas competencias presentes en el currículo español se compone de diferentes dimensiones que las describen en forma más específica, por lo que, a partir de ellas, se presentará cómo se vinculan con la educación física y, en especial, con la dimensión emocional.

La competencia lingüística contiene entre sus dimensiones el elemento de la comunicación, ya sea de carácter verbal o no verbal, por lo que a través de la práctica de actividad física y deporte, se debe promover una adecuada comunicación que permita tanto 
a los docentes como al alumnado transmitir ideas y sentimientos (Braz \& Coral, 2016). Otra de las dimensiones de esta competencia hace referencia a la autorregulación de las emociones y del comportamiento, teniendo como principal objetivo, "facilitar el propio aprendizaje, adoptar decisiones que ajusten el propio comportamiento motor y/o emocional y saber explicarlas" (Braz \& Coral, 2016, p. 54).

Las competencias básicas en ciencia y tecnología presentan una dimensión llamada la protección de la salud individual y colectiva, la cual hace énfasis en el desarrollo de la consciencia corporal en las diferentes tareas educativas, como la consciencia de las emociones, de la respiración o de la musculatura (Braz \& Coral, 2016, p. 54). La competencia social y cívica tiene entre sus dimensiones la adquisición de habilidades sociales, que promueven el desarrollo moral del alumnado en las actividades físico-deportivas de la clase de educación física. Otra dimensión es la creación de un sistema de valores propio, lo cual "ayudará al alumnado a reflexionar sobre las propias actitudes respecto a la práctica de actividad física, reconociendo emociones y salvando los dilemas que surjan no solo en el ejercicio de una práctica deportiva, sino también en su vida cotidiana" (Braz \& Coral, 2016, p. 58).

En la competencia aprender a aprender se encuentra una dimensión sobre la consciencia de las propias actividades, la cual hace referencia al reconocimiento de las fortalezas y debilidades personales con respecto al punto de partida con la práctica motriz (Braz \& Coral, 2016). Esta dimensión es fundamental al momento de enfrentarse a una unidad didáctica sobre un deporte en el que el alumnado posee experiencias deportivas de buen nivel, como por ejemplo, que hayan sido adquiridas en clubes deportivos. Otra dimensión es la del sentimiento de competencia personal, la cual ayudará a los estudiantes a "establecer metas alcanzables, a proporcionar autoconfianza, a saber dosificar el esfuerzo, a aceptar los propios errores de forma constructiva y a aprender de y con los demás” (Braz \& Coral, 2016, p. 60).

Finalmente, la última competencia, sentido de la iniciativa y espíritu emprendedor, tiene como dimensión el desarrollo personal, en la cual se le plantea al alumnado la "necesidad de autosuperarse, perseverar y adoptar una actitud positiva ante tareas 
de cierta dificultad técnica o en la mejora del nivel de la condición física, buscando soluciones y siendo constante en la práctica de la actividad física" (Braz \& Coral, 2016, p. 60). Con respecto a la relación que tendrían las competencias mencionadas con la unidad didáctica, González y Campos (2014) mencionan que el alumnado debe adquirir al término de la educación secundaria obligatoria cada una de las competencias, por lo que, durante todo ese proceso, el profesorado debe justificar qué aspecto de ellas se está desarrollando con las actividades realizadas en las diferentes programaciones de la clase de educación física.

Entre los aspectos que destaca Gallardo (2016) para facilitar el proceso de adquisición de las competencias se encuentran los siguientes: a) contextualizar los procesos de enseñanza-aprendizaje, b) ofrecer funcionalidad, c) implementar situaciones transferibles a la vida cotidiana, d) globalizar los aprendizajes, e) potenciar la interacción entre iguales, f) ofrecer diversas formas de organización social del aula y g) implementar métodos activos. Una de las metodologías activas que se ha propuesto para la educación física con base en competencias corresponde al trabajo cooperativo que puede desarrollarse a través de proyectos, el cual sería reconocido por favorecer una buena disposición en el alumnado para las tareas motrices, ya que genera "una influencia positiva en las relaciones interpersonales favorece un clima positivo en el grupo, promueve autoconceptos positivos y mejora la motivación hacia los aprendizajes, además de constituir un excelente recurso para fomentar un clima positivo que promueve la inclusión" (Gallardo, 2016, p. 102).

\section{Educación física en Chile}

El currículo de educación física en Chile ha sido criticado por inclinarse hacia una educación de carácter biomédico, que otorga una concepción mecanicista al cuerpo humano, contraria a los actuales modelos que asumen un carácter más crítico y menos dualista desde la perspectiva cartesiana en torno al cuerpo humano (Moreno, 2018; Moreno, Gamboa \& Poblete, 2014). Por ello, también se ha señalado que en la actualidad la educación física chilena tiene "una fuerte tendencia a medicalizar y perder el sentido educativo y de juego como ha sido concebido a través del tiempo" (Cornejo \& Matus, 2013, p. 23). En consecuencia, la calidad de la educación física en 
el país fue reducida a una evaluación nacional de la condición física y de factores antropométricos del alumnado de octavo año básico (Agencia de la Calidad de la Educación, 2016), que ignora los factores morales, afectivos y psicosociales que forman parte de la asignatura y son esenciales en una concepción constructivista e integrada de la educación (Mujica et al., 2017). Además, es preciso señalar que esta prueba evaluación nacional "no tiene una relación acertada con la distribución de los contenidos estipulados por los planes y programas de Educación Física” (Rodríguez-Rodríguez, Curilem, Escobar \& Valenzuela, 2016). En síntesis, esta prueba estandarizada excluye importantes contenidos del currículo de educación física en Chile (Rodríguez et al., 2015).

Sin embargo, ha de destacarse que los nuevos programas curriculares de esta asignatura han enfatizado en un concepto importante para la educación constructivista, que son las actitudes del alumnado, interpretadas por el organismo educativo del país como un factor importante en la formación de ciudadanos responsables y participativos en los temas contingentes de la sociedad (Ministerio de Educación, 2016). Así mismo, en el documento curricular de la asignatura explican que las actitudes están compuestas por la dimensión afectiva, la cual "corresponde a los sentimientos que un objeto suscita en los individuos" (Ministerio de Educación, 2016, p. 12).

Un estudio en el que participaron estudiantes de enseñanza básica en Chile abordó la percepción de las actitudes del profesorado de educación física y concluyó que por medio de las sesiones se reproducen estereotipos de género en relación con la práctica deportiva, no obstante, existe una valoración más positiva que negativa sobre la actuación docente (Gálvez et al., 2016). Un segundo estudio realizado también en enseñanza básica logró valorar la percepción emocional del alumnado en las actividades orientadas al desarrollo de la condición física e identificó ocho atribuciones causales de las emociones positivas para el bienestar subjetivo y trece atribuciones causales de las emociones negativas para el bienestar subjetivo (Mujica, Orellana, Aránguiz \& González, 2016). Esto demuestra que en estas actividades que demandan el esfuerzo del alumnado se experimentan muy diversas emociones, tanto positivas como negativas, de modo que no solo en las actividades lúdicas el alumnado percibe emociones agradables o positivas para su bienestar. 
Desde una perspectiva constructivista, significa un gran avance que el profesorado pueda atender e investigar pedagógicamente las emociones de su alumnado, ya que este conocimiento puede ser aprovechado para propiciar las actitudes positivas de los estudiantes ante los diferentes contenidos. No obstante, la presencia de este contenido en el currículo no asegura que el profesorado le otorgue el tiempo suficiente a este asunto, ya que los objetivos actitudinales emergen de los objetivos de aprendizaje transversal [OAT] y el currículo manifiesta que deben ser promovidos, pero no evaluados en un proceso sistemático. Por consiguiente, ante la poca cantidad de horas que tiene la asignatura, es comprensible que los docentes le otorguen la mayor cantidad del tiempo a los objetivos de aprendizaje verticales [OAV] que serán evaluados y calificados, los cuales se asocian a las unidades didácticas en torno a tres ejes curriculares: a) habilidades motrices, b) vida activa y saludable, y c) responsabilidad personal y social en el deporte y la actividad física.

Como se acaba de mencionar, el problema que se identifica de los OAT y sus objetivos actitudinales es la forma de operacionalizar estos fines educativos tan importantes para la educación integral de los escolares del país. En este modelo curricular es posible identificar una idea implícita que sostiene un imposible fracaso en el logro de la educación transversal en el transcurso de la formación escolar. Debido a que es requisito por ley asegurar una educación integral en el país, sería ilógico pensar que el currículo establece procesos pedagógicos donde no importa que los estudiantes no aprendan lo que se dice enseñar, por lo tanto, es coherente que el currículo se encuentre diseñado para asegurar por lo menos un mínimo de esta formación transversal. No obstante, al estudiar su estructura dentro del currículo, se observa que no existe una sistematización de estos aprendizajes y se carece de evidencias obtenidas por evaluaciones rigurosas y regulares que indiquen a los estudiantes y a sus familias el progreso cuantitativo y cualitativo en su formación integral. Esta situación, que desvalora y superficializa la transversalidad, queda demostrada en la promoción de los educandos, la cual está enfocada solo en los OAV, referidos a conocimientos y habilidades. Ante este panorama curricular, se aprecia la necesidad de repensar la educación integral en el sistema educativo escolar chileno, ya que "comprender el emocionar del alumnado es una tarea compleja, que requiere construir condiciones pedagógicas que se basen en 
la ética y en la empatía profesional" (Mujica, 2018). De modo que, si se desea avanzar en la perspectiva constructivista, se debe en lo posible incorporar elementos curriculares que le otorguen una mayor rigurosidad al desarrollo afectivo, como lo son actividades didácticas, estándares de aprendizaje, criterios de evaluación e instrumentos de evaluación.

\section{Conclusión}

Considerando la interacción entre las personas y la cultura, por medio de la cual se produce la construcción de la identidad, es de gran relevancia que el currículo de la asignatura de educación física garantice en el proceso pedagógico la responsabilidad con la dimensión afectiva-emocional del alumnado, la cual debe ser abordada por el profesorado desde una perspectiva comprensiva con la finalidad de contribuir al desarrollo de una buena actitud con los contenidos de aprendizaje, más aún cuando existan elementos que pueden estar afectando negativamente la participación y la motivación de los estudiantes. Además, es fundamental obtener y triangular antecedentes sobre las experiencias familiares, recreativas y/o deportivas que definen el entorno sociocultural del alumnado, las cuales pueden ser de vital utilidad para resolver asuntos prácticos en torno a la dimensión afectiva de los educandos.

En cuanto al modelo curricular de los dos países analizados, se aprecia que el currículo chileno debe abordar con mayor rigurosidad el aprendizaje transversal del alumnado, para de esta forma responder con una evaluación sistemática al progreso de la formación integral. De lo contrario, se estaría realizando una educación constructivista solo desde un plano declarativo.

Por último, cabe mencionar que cada nación tiene sus propias características, condiciones y problemáticas socioculturales, por lo que la investigación pedagógica en Chile y Latinoamérica en torno a las emociones en la asignatura debería ser más fomentada. Asimismo, este proceso requiere la suficiente calidad para que pueda ser parte de una publicación científica, ya que de esta forma podría contribuir en mayor grado a la discusión académica y curricular de la región que, evidentemente, requiere progresar en la formación integral de la ciudadanía. 


\section{Referencias}

Agencia de la Calidad de la Educación. (2016). Informe resultados estudio nacional de Educación Física. Santiago: ACE.

Albornoz, Y. (2009). Emoción, música y aprendizaje significativo. Revista Educere, 13(44), 67-73.

Bermúdez, C., \& Sáenz-López, P. (2019). Emociones en Educación Física. Una revisión bibliográfica (2015-2017). Retos. Nuevas Tendencias en Educación Física, Deporte y Recreación, 36, 597-603.

Blández, J. (2000). Programación de unidades didácticas según ambientes de aprendizaje. Barcelona: Inde.

Boletín Oficial del Estado. (2014). Real Decreto 126/2014, de 28 de febrero, por el que se establece el currículo básico de la Educación Primaria. Madrid: Ministerio de Educación, Cultura y Deporte.

Bolívar, A. (1992). Los contenidos actitudinales en el currículo de la reforma. Problemas y respuestas. Madrid: Escuela Española.

Braz, M., \& Coral, J. (2016). ¿Qué tiene que ver la educación física con las competencias? En T. Lleidà, \& E. Sebastiani (Eds.), Competencias clave y Educación Física (pp. 47-62). Barcelona: Inde.

Bruner, J. (1989). Acción, pensamiento y lenguaje. Madrid: Alianza.

Bruner, J. (1991). Actos de significado. Más allá de la revolución cognitiva. Madrid: Alianza.

Bruner, J. (1997). La educación, puerta de la cultura. Madrid: Visor.

Cabalé, E., \& Rodríguez, G. (2017). Educación no formal: potencialidades y valor social. Revista Cubana de Educación Superior, 36(1), 69-83.

Cáceres, Z., \& Munévar, O. (2016). Evolución de las teorías cognitivas y sus aportes a la educación. Revista Actividad Física y Desarrollo Humano, 7, 1-13.

Capitán, A. (2002). Breve historia de la educación en España. Madrid: Alianza.

Carr, W., \& Kemmis, S. (1988). Teoría crítica de la enseñanza. Barcelona: Martínez Roca. 
Castejón, F. (2010). Deporte como concepto y aplicación. En F. Castejón (Ed.), Deporte y Enseñanza Comprensiva (pp. 11-34). Sevilla: Wanceulen.

Colom, A. (2005). Continuidad y complementariedad. Entre la educación formal y no formal. Revista de Educación, 338, 9-22.

Comisión Europea/EACEA/Eurydice. (2013). La educación física y el deporte en los centros escolares de Europa. Informe de Eurycide. Luxemburgo: Oficina de Publicaciones de la Unión Europea.

Cornejo, M., \& Matus, C. (2013). Educación Física en Chile. Actividad Física y Ciencias, 15(1), 1-25.

Duran, C., Lavega, P., Planas, A., Muñoz, R., \& Pubill, G. (2014). Educación física emocional en secundaria. El papel de la sociomotricidad. Apunts. Educación Física y Deportes, 117(3), 23-32. https://dx.doi. org/10.5672/apunts.2014-0983.es(2014/3).117.02

Duran, C., Lavega, P., Salas, C., Cristofol, T., Tamarit, M., \& Invernó, J. (2015). Educación física emocional en adolescentes. Identificación de variables predictivas de la vivencia. Cultura, Ciencia y Deporte, $10(28), 5-18$.

Echeita, G., \& Martín, E. (1990). Interacción social y aprendizaje. En A. Marchesi, C. Coll, \& J. Palacios (Eds.), Desarrollo psicológico y educación (pp. 49-67). Madrid: Alianza.

Gallardo, S. (2016). ¿Cómo se enseñan las competencias? En T. Lleidà, \& E. Sebastiani (Eds.), Competencias clave y Educación Física (pp. 91108). Barcelona: Inde.

Gálvez, C., Maureira, F., Flores, E., Véliz, C., Cea, S., Soto, C., \& Soto, H. (2016). Percepción de los estudiantes de primaria hacia sus profesores de Educación Física en Santiago de Chile. EmásF. Revista Digital de Educación Física, 41, 133-142.

Gambau, V. (2015). Las problemáticas actuales de la Educación Física y el Deporte escolar en España. Revista Española de Educación Física y Deportes, 411, 53-69.

Germania, N., \& García, C. (2017). Autoestima, educación emocional y su incidencia en el proceso de enseñanza aprendizaje de los estudiantes en las instituciones educativas. Boletín Redipe, 6(5), 84-92. 
Gil, P., \& Martínez, M. (2016). Emociones percibidas, por alumnos y maestros, en Educación Física en $6^{\circ}$ curso de primaria. Educación XX1, 19(2), 179-204. https://doi.org/10.5944/educXX1.14230

González, F. (2009). La significación de Vygotski para la consideración de lo afectivo en la Educación: Las bases para la cuestión de la subjetividad. Revista Electrónica "Actualidades Investigativas en Educación”, 9, 1-24.https://doi.org/10.15517/AIE.V9I4.9519

González, M., \& Campos, A. (2014). Intervención Docente en Educación Física en Secundaria y en el Deporte Escolar. Madrid: Síntesis.

Jordi, J., \& Torrebadella, X. (2018). Reflexiones para (re)formular una educación física crítica. Revista Internacional de Medicina y Ciencias de la Actividad Física y el Deporte, 18(71), 441-462.https://dx.doi. org/10.153366/rimcafd2018.71.003

Lacasa, P. (1994). Aprender en la escuela, aprender en la calle. Madrid: Visor.

Lleidà, T. (2016). ¿Qué es realmente esto de las competencias?. En T. Lleidà, \& E. Sebastiani (Eds.), Competencias clave y Educación Física (pp. 19-30). Barcelona: Inde.

López, V. (2010). Perspectiva constructivista del aprendizaje y de la enseñanza del deporte. En F. Castejón (Ed.), Deporte y Enseñanza Comprensiva (pp. 35-62). Sevilla: Wanceulen.

López-Ros, V., Pradas, R., \& Font, R. (2014). Educación Física en primaria. Desarrollo curricular y estrategias para la práctica docente. Barcelona: Universitat de Barcelona.

Méndez, D., Fernández-Río, J., Méndez, A., \& Prieto, J. (2015). Análisis de los currículos autonómicos LOMCE de Educación Física en Educación Primaria. Retos. Nuevas Tendencias en Educación Física, Deporte y Recreación, 28, 15-20.

Ministerio de Educación. (2016). Educación Física y Salud. Programa de estudio primer año medio. Santiago: Chile.

Moreno, A. (2018). La Educación Física chilena en educación básica: Una caracterización crítica. Journal of the Latin American socio-cultural studies of sport, 9(2), 65-78. https://dx.doi.org/10.5380/jlasss. v9i2.61261 
Moreno, A., Gamboa, R., \& Poblete, C. (2014). La educación física en Chile: Análisis crítico de la documentación ministerial. Revista Brasileira de Ciências do Esporte, 36(2), 411-427. https://dx.doi.org/10.159o/ So101-32892014000200010

Moreno, A., Rivera, E., \& Trigueros, C. (2014). Sistema de medición de la calidad de la Educación Física Chilena: un análisis crítico. Movimento, 2O(1), 145-167. https://doi.org/10.22456/8918.41485

Mujica, F., Orellana, N., Aránguiz, H., \& González, H. (2016). Atribución emocional de escolares de sexto año básico en la asignatura de Educación Física y Salud. Educación Física y Ciencia, 18(2), 1-6.

Mujica, F., Orellana, N., \& Concha, R. (2017). Emociones en la clase de Educación Física: revisión narrativa (2010-2016). Revista Ágora para la Educación Física y el Deporte, 19(1), 119-134.

Mujica, F. (2018). Las emociones en la educación física escolar. El aporte de la evaluación cualitativa. EmásF. Revista Digital de Educación Física, 51, 64-78.

Mujica-Johnson, F., \& Jiménez, A. (2019). Percepción emocional en la asignatura de Baloncesto de estudiantes del Grado en Ciencias del Deporte: Estudio piloto. Cuadernos de Psicología del Deporte, 19(2), 152-166

Mula, A., \& Navas, L. (2011). Las actitudes ante la inmigración en los adolescentes y en los jóvenes. Alicante: Club Universitario.

Pastor, J., \& Cuevas, R. (2011). Las competencias básicas en Educación Física: Programación y diseño de tareas. En O. Contreras, \& R. Cuevas (Ed.), Las competencias básicas desde la Educación Física (pp. 4562). Barcelona: Inde.

Perales, F. (2000). Didáctica de las ciencias experimentales. En L. Rico, \& D. Madrid (Eds.), Fundamentos didácticos de las áreas curriculares (pp. 17-64). Madrid: Síntesis.

Pérez, Á. (1999). Las funciones sociales de la escuela: De la reproducción a la reconstrucción crítica del conocimiento y la experiencia. En J. Gimeno, \& Á. Pérez (Ed.), Comprender y transformar la enseñanza (pp. 17-33). Madrid: Morata.

Rivas, J., Leite, A., \& Cortés, P. (2014). Formación del profesorado y experiencia escolar: las historias de vida como práctica educativa. Praxis Educativa, 18(2), 13-23. 
Rodríguez, F., Estrada, D., Quezada, T., Tapia, A., Valderrama, C., \& Moreno, A. (2015). Sistema de medición de la calidad de la educación física en Chile y su influencia en la realidad escolar. Movimento, 21(2), 435-448.

Rodríguez-Rodríguez, F., Curilem, C., Escobar, D., \& Valenzuela, L. (2016). Propuesta de evaluación de la educación física escolar en Chile. Educación Física y Ciencia, 18(1), 1-12.

Sarramona, J. (2008). Teoría de la educación. Barcelona: Ariel.

Vicente, M. (2016). Bases per a una didáctica crítica de l'educació física. Apunts. Educació Física i Esports, 123, 76-85. http://dx.doi. org/10.5672/apunts.2014-0983.cat.(2016/1).123.09

Viera, T. (2003). El aprendizaje verbal significativo de Ausubel. Algunas consideraciones desde el enfoque histórico-cultural. Revista Universidades, 26, 37-43.

Vygotsky, L. (1979). El desarrollo de los procesos psicológicos superiores. Barcelona: Crítica.

Vygotsky, L. (2000). Psicologia concreta do homen. Educação \& Sociedade, 21(71), 21-44.

Zamorano, M., Gil-Madrona, P., Prieto-Ayuso, A., \& Zamorano, D. (2018). Emociones generadas por distintos tipos de juegos en clase de educación física. Revista Internacional de Medicina y Ciencias de la Actividad Física y el Deporte, 18(69), 1-26. https://doi.org/10.15366/ rimcafd2018.69.001 